\title{
Supporting Everyday Thinking Practices in Information Visualization Interfaces
}

Jagoda Walny

Innovis Group, Interactions Lab

University of Calgary

Calgary, AB, Canada

jkwalny@ucalgary.ca

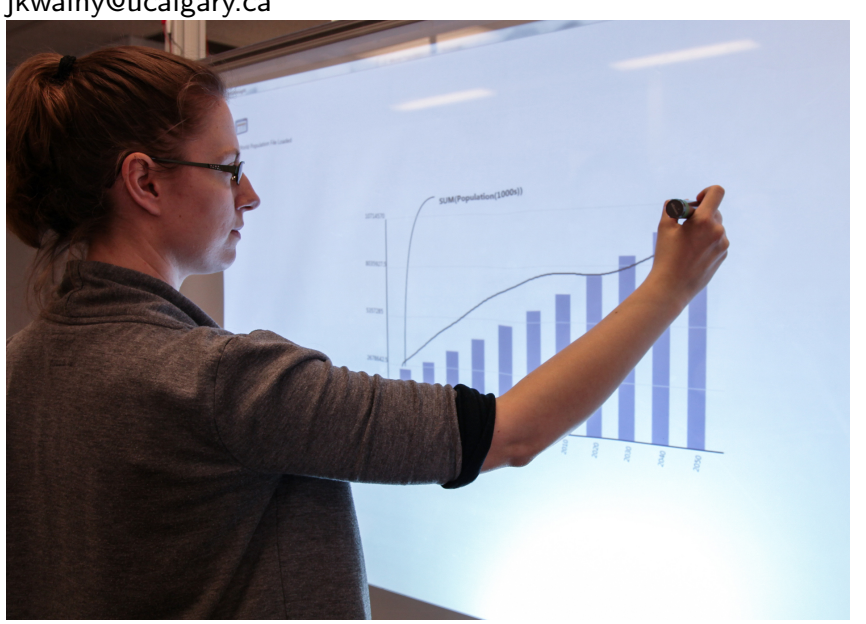

Figure 1: Changing a bar chart into a line chart using the prototype created for our Wizard of Oz study [19] for understanding how people would approach interactively sketching visualizations.

Permission to make digital or hard copies of part or all of this work for personal or classroom use is granted without fee provided that copies are not made or distributed for profit or commercial advantage and that copies bear this notice and the full citation on the first page. Copyrights for third-party components of this work must be honored. For all other uses, contact the owner/author(s). Copyright is held by the author/owner(s).

ITS 2014, November 16-19, 2014, Dresden, Germany.

ACM 978-1-4503-2587-5/14/11.

http://dx.doi.org/10.1145/2669485.2669491

\begin{abstract}
People commonly sketch externalizations on paper and whiteboards as part of their everyday thinking processes. While common, this practice is little understood, particularly as it may relate to digital visual representation (such as information visualization) and sketch-based interaction. My research aims to better understand these thinking sketches from the perspective of information visualization and pen-and-touch interaction and to apply this understanding to the design of interfaces that can better support complex and freeform everyday thinking practices.
\end{abstract}

\section{Author Keywords}

information visualization; pen-and-touch interaction; sketched externalizations; qualitative evaluation

\section{ACM Classification Keywords}

H.5.2 [Information interfaces and presentation (e.g., $\mathrm{HCl})$ ]: User Interfaces.

\section{Introduction}

When visiting the offices of knowledge workers, it is not uncommon to see diagrams, mind maps, and scraps of information scribbled on whiteboards alongside notebooks filled with ideas and sketches for problem solving. These visual artifacts are residue of the process of externalization 
- the reification of internal thought to support cognition. Externalizations support cognition in a variety of ways, including providing a way to offload memory or to solve problems more efficiently [8]. They are often ad-hoc, created on the fly to suit a given context. This spontaneous practice becomes more difficult when the objects of thought are dynamic representations of digital data that would be tedious to reproduce manually but whose manipulation is restricted by the design of the interface containing it. The availability of pen and touch hardware provides an opportunity to better support such thinking practices with digital information.

The goal of my research is to further our understanding of how to better support externalization for digital

information. The information visualization community has tackled its goal of aiding cognition of digital information

[5] by shaping data into more manageable and interactive representations. The approaches to this have been mainly data-centric [14] (shaped by the data) or task-centric [1] (shaped by the tasks to be accomplished with the data). However, while these approaches make the information or select aspects of the information - easier to understand, this still leaves room for a complementary human-centric approach, in which people's common thinking processes are supported.

My broad approach to this research consists of two phases:

- 1. Exploration: Deepen our understanding of externalization from an information visualization perspective by performing qualitative exploratory studies. In particular, I look at sketched externalizations.

- 2. Application: Apply this deepened understanding to the design of interfaces and/or interaction techniques that support externalization, with a particular focus on using interactive pen- and touch-enabled surfaces.

In my research, I aim to leverage the similarity of pen and touch surfaces to conventional whiteboards to support thinking with digital information. As such, I build on prior work on pen and touch interfaces as well as work done on supporting paper- or whiteboard-based practices digitally. Sketch-based interfaces date back to Sutherland's Sketchpad [15], and have been used, among others, for modeling 3D objects [12], for interactive mathematical sketching $[2,9,10]$, for creating visual queries of data $[20,13]$, and creating information visualizations [6, 4]. Typically, in these systems, sketches are interpreted as commands or inputs to the system. In contrast, my research is concerned with creating interfaces that support freeform sketched externalizations, which may not always have a pre-defined or expected form that can be interpreted. Pen and touch interfaces provide a richer potential set of tools [7], and recent work suggests that bimanual pen and touch interactions can be effective [3].

Prior research into supporting knowledge workers' thinking practices in general has resulted in systems that emulate and extend conventional whiteboards with the advantages of the digital environment [11]. My focus is not on creating digital whiteboards, but on supporting the kinds of behaviours one finds on conventional whiteboards for those working with digital information. One approach that allows for some externalization practices when working with digital information has involved integrating digital and analog technologies. For example, Brandl et al. created NiCEBook [3], a paper notebook that synchronizes with its digital representation. This approach has the advantage of preserving the affordances of paper, 
but may not be sufficient when working with dynamic visualizations of data.

\section{Exploratory Studies}

I have completed three qualitative exploratory studies of externalization through sketching, summarized here.

\section{Visualizations on Whiteboards}

To arrive at a better understanding of the nature of visuals created spontaneously during everyday thinking tasks, we studied a collection of 82 snapshots of 69 knowledge workers' whiteboards collected at a large research institution [17]. As these whiteboards were in individuals' offices, they contained the residue of various kinds of thought, from small pieces of information to remember, to sketches used to work out problems, to those used in small-group discussions. We analyzed these qualitatively from an information visualization perspective, examining the types of visual constructs used (both recognizable and novel) as well as the relationship between words and diagrammatic constructs. Follow-up interviews with a subset of the participants validated our categorization of constructs and gave us valuable insight into the nature of the visuals.

This study resulted in a taxonomy of the kinds of constructs and visual strategies people use to express their thoughts, including communicative symbols, layering, spatial organization, and information visualization constructs. It also demonstrated the words-to-diagrams spectrum, which describes the variable nature of how words are used in relation to diagrammatic constructs. This highlighted the restrictive nature of software that, when providing support for making diagrams or visualizations, considers words only in the role of labels.

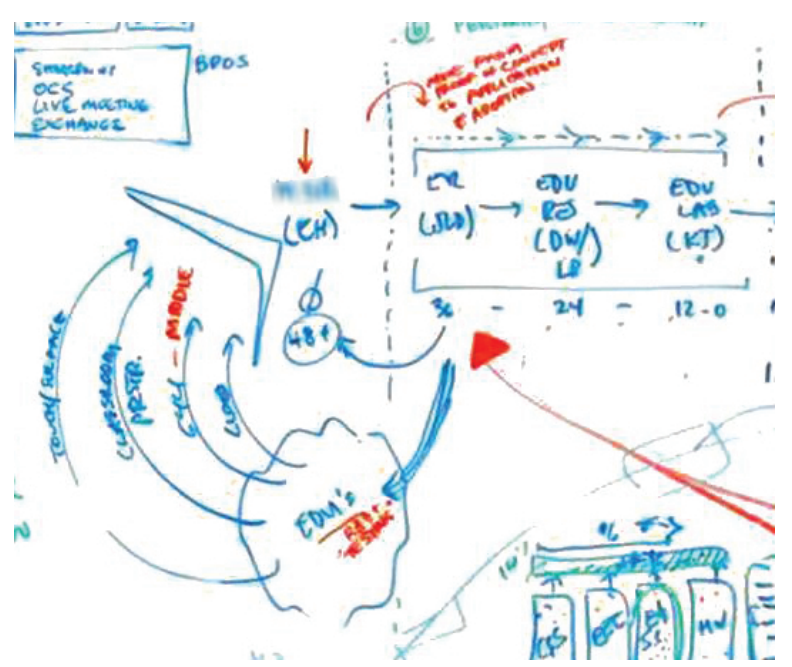

Figure 2: An example of a complex, freeform externalization on a whiteboard. (C)2011 IEEE. Reprinted, with permission, from [17].

\section{Sketching Data}

Sketching concepts and ideas on whiteboards may not be entirely analogous to sketching data. We asked people to use colored pencils to sketch a small, relatable, and easily understandable dataset in whatever way they chose and to report on what they learned about the data [16]. Our analysis has focused on qualitatively classifying the wide variety of representations collected and relating the types of representations to the types of insights gained.

Lifecycles of Diagrams and Sketches

We have also examined the context of externalization practices on a broader level by interviewing computer scientists about sketches and diagrams that were important to a recent project [18]. From these interviews, we reconstructed the lifecycles of these sketches in terms 
of the context (personal, group, or public), the medium used (e.g. paper, tablet, whiteboard), and the transitions that a sketch underwent (e.g. creation, refinement, sharing). See Figure 3 for a sample. This study highlighted the variety of habits and strategies used by people in their thinking processes, suggesting that a one-size-fits-all interface for supporting thinking may not be feasible.

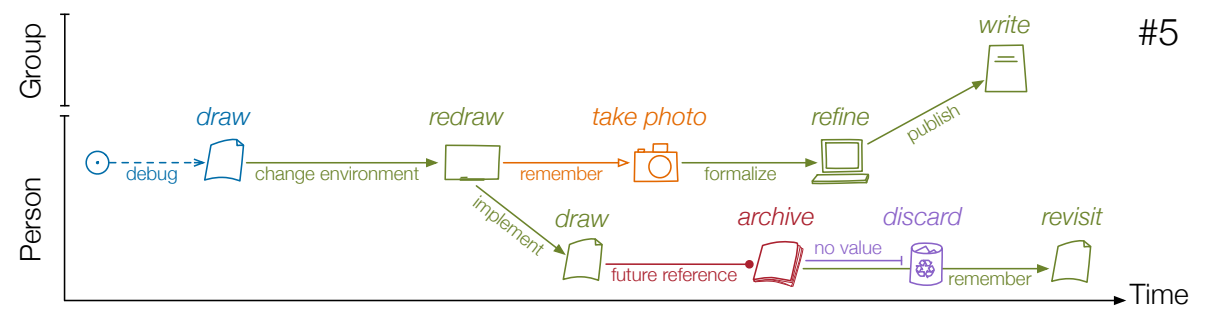

Figure 3: A lifecycle from of a single sketch significant to a computer scientist's project. (C)2011 IEEE. Reprinted, with permission, from [18].

\section{Studying Application Challenges}

This phase of my research involves discovering how the insights gained in the exploratory studies can be applied to information visualization tools that support externalization. The purpose of this is to highlight the challenges inherent in the design of such tools and to suggest some design strategies moving forward. As such, my plan is to use as a basis standard interactive visualizations typical of what is commonly seen on the web, such as interactive bar charts and graph visualizations. I am focused in particular on the context of pen-and-touch surfaces due to their being the closest in nature to an analog whiteboard or piece of paper. To date, we have completed one study; the next steps are described in the following section.
Understanding Pen and Touch Interaction for Data Exploration

Our first foray into designing an interactive system that supports thinking practices was to design a system for creating simple visualizations based on real data just by sketching them [19]. We ran a Wizard of Oz study based on our design in order to see how people would approach interactively sketching a simple information visualization. During the study, we asked participants to figure out how to use the system on their own in order to see what types of interactions they tended towards naturally.

Our system, shown in Figure 1, was designed to keep the interface out of the way as much as possible by supporting specification of what one wants to see by simply drawing it, supporting manipulation of objects that is as direct as possible, minimizing explicit mode-switching, and providing good default behaviours.

Our study results indicated that participants drew on knowledge from both the physical world and from what they had already learned about the system in order to infer how to perform new tasks. Our participants had a clear idea of when to use pen and when to use multitouch interactions, which was drawn from their experience in the physical world; they also used a variety of postures when holding the pen and performing combined pen and touch interactions. They used multiple interaction paths for the same task and also tended to perform integrated interactions, where interaction with the data and other elements on the screen occurs in proximity to the objects being acted upon.

\section{The Next Steps}

My initial inquiry into applying the results of the above studies to information visualization software has 
highlighted some of the particular challenges of designing thinking interfaces. The design of these interfaces goes beyond usability and learnability into supporting complex and often freeform and rapid thinking practices. One challenge is in knowing how to create interfaces that do not interfere with thought processes. Another challenge is in supporting externalization practices for dynamic representations of information. My current project, following from our study of whiteboards [17], explores the space where words and diagrammatic elements are tightly intermingled in a single thinking diagram. This poses several interesting design challenges such as knowing when to distinguish between words and drawn elements.

The exploratory portion of my research is largely complete, and my aim is to use the remainder of my $\mathrm{PhD}$ to apply the understanding I have gained through these studies to the creation of software, particularly as mentioned above, making use of the words-to-diagrams spectrum. However, I believe that an important part of my $\mathrm{PhD}$ contribution will remain the new understanding of how people use sketched visuals as externalizations during their thinking processes.

\section{Acknowledgements}

I would like to thank my supervisor, Sheelagh Carpendale, and my collaborators and colleagues in both the Interactions Lab and Microsoft Research for their support. This research was supported in part by Microsoft Research, NSERC, SMART Technologies, Alberta Innovates Technology Futures, SurfNet, and GRAND.

\section{References}

[1] Amar, R., and Stasko, J. A knowledge task-based framework for design and evaluation of information visualizations. In Information Visualization, IEEE Symposium on (2004), 143-150.

[2] Bott, J. N., and LaViola, Jr., J. J. A pen-based tool for visualizing vector mathematics. In Proceedings of the Seventh Sketch-Based Interfaces and Modeling Symposium, SBIM '10, Eurographics Association (Aire-la-Ville, Switzerland, Switzerland, 2010), 103-110.

[3] Brandl, P., Forlines, C., Wigdor, D., Haller, M., and Shen, $C$. Combining and measuring the benefits of bimanual pen and direct-touch interaction on horizontal interfaces. In Proceedings of the Working Conference on Advanced Visual Interfaces, AVI '08, ACM (New York, NY, USA, 2008), 154-161.

[4] Browne, J., Lee, B., Carpendale, S., Riche, N., and Sherwood, T. Data analysis on interactive whiteboards through sketch-based interaction. In Proceedings of the ACM International Conference on Interactive Tabletops and Surfaces, ITS '11, ACM (New York, NY, USA, 2011), 154-157.

[5] Card, S. K., Mackinlay, J. D., and Shneiderman, B. Readings in information visualization: Using vision to think. Morgan Kaufmann, 1999.

[6] Chao, W. O., Munzner, T., and van de Panne, M. Poster: Rapid pen-centric authoring of improvisational visualizations with NapkinVis. Posters Compendium InfoVis (2010).

[7] Hinckley, K., Yatani, K., Pahud, M., Coddington, N., Rodenhouse, J., Wilson, A., Benko, H., and Buxton, B. Pen + touch $=$ new tools. In Proceedings of the 23rd Annual ACM Symposium on User Interface Software and Technology, UIST '10, ACM (New York, NY, USA, 2010), 27-36.

[8] Kirsh, D. Thinking with external representations. Al Society 25, 4 (2010), 441-454. 
[9] LaViola, Jr., J. J., and Zeleznik, R. C. Mathpad2: A system for the creation and exploration of mathematical sketches. In ACM SIGGRAPH 2007 Courses, SIGGRAPH '07, ACM (New York, NY, USA, 2007)

[10] Leitner, J., Rendl, C., Perteneder, F., Gokcezade, A., Seifried, T., Haller, M., Zeleznik, R., and Bragdon, A. NiCE formula editor. In ACM SIGGRAPH 2010 Talks, SIGGRAPH '10, ACM (New York, NY, USA, 2010), 55:1-55:1.

[11] Mynatt, E., Huang, E., Voida, S., and Maclntyre, B. Large displays for knowledge work. In Public and Situated Displays, K. OHara, M. Perry, E. Churchill, and D. Russell, Eds., vol. 2 of The Kluwer International series on Computer Supported Cooperative Work. Springer Netherlands, 2003, 80-102.

[12] Olsen, L., Samavati, F. F., Sousa, M. C., and Jorge, J. A. Sketch-based modeling: A survey. Computers \& Graphics 33, 1 (2009), 85-103.

[13] Ryall, K., Lesh, N., Lanning, T., Leigh, D., Miyashita, H., and Makino, S. Querylines: Approximate query for visual browsing. In $\mathrm{CHI}$ '05 Extended Abstracts on Human Factors in Computing Systems, CHI EA '05, ACM (New York, NY, USA, 2005), 1765-1768.

[14] Shneiderman, B. The eyes have it: A task by data type taxonomy for information visualizations. In
Visual Languages, IEEE Symposium on (1996), 336-343.

[15] Sutherland, I. E. Sketch pad: A man-machine graphical communication system. In Proceedings of the SHARE Design Automation Workshop, DAC '64, ACM (New York, NY, USA, 1964), 6.329-6.346.

[16] Walny, J., and Carpendale, S. Data sketches: An exploratory study. InfoVis 2014 Posters Compendium (to appear).

[17] Walny, J., Carpendale, S., Riche, N., Venolia, G., and Fawcett, P. Visual thinking in action: Visualizations as used on whiteboards. Visualization and Computer Graphics, IEEE Transactions on 17, 12 (Dec 2011), 2508-2517.

[18] Walny, J., Haber, J., Dork, M., Sillito, J., and Carpendale, S. Follow that sketch: Lifecycles of diagrams and sketches in software development. In Visualizing Software for Understanding and Analysis (VISSOFT), 2011 6th IEEE International Workshop on (Sept 2011), 1-8.

[19] Walny, J., Lee, B., Johns, P., Riche, N., and Carpendale, S. Understanding pen and touch interaction for data exploration on interactive whiteboards. Visualization and Computer Graphics, IEEE Transactions on 18, 12 (Dec 2012), 2779-2788.

[20] Wattenberg, M. Sketching a graph to query a time-series database. In CHI '01 Extended Abstracts on Human Factors in Computing Systems, CHI EA '01, ACM (New York, NY, USA, 2001), 381-382. 